\title{
Novel Phosphorus Radical-based Routes to Horsfiline
}

John A. Murphy*, Régis Tripoli, Tanweer A. Khan and Umesh W. Mali

WestCHEM, Department of Pure and Applied Chemistry, University of Strathclyde, 295 Cathedral Street, Glasgow G1 1XL, United Kingdom.

Email Address john.murphy@strath.ac.uk 


\section{Index}

Experiment Page

1-tert-\{(Butyloxy)carbonyl\}-3-(methyloxycarbonyl)pyrrolidine 3

Pyrrolidine-1,3-dicarboxylic acid 1-tert-butyl ester 3

Benzyl 1-tert-\{butyloxycarbonyl\}pyrrolidine-3-carboxylate 10a 4

$\mathrm{N}$-2-Iodo-4-methoxyphenyl-1-tert-\{butyloxycarbonyl\}pyrrolidine-3-carboxamide 12a 4

$N$-(Trimethylsilyl)ethoxymethyl- $N$-[2-iodo-4-methoxyphenyl]-1-tert-\{butyloxy-

carbonyl \}-pyrrolidine-3-carboxamide 12b

5-Methoxy-2-oxo-1-(2-trimethylsilanylethoxymethyl)-1,2-dihydrospiro[indole-

3,3'-pyrrolidine]-1'-carboxylic acid tert-butyl ester 13 and 5-methoxy- $N$-(trimethyl-

silyl)ethoxymethyl- $N$-phenyl-1-tert-\{butyloxycarbonyl $\}$-pyrrolidine-3-carboxamide 14a

5-Methoxy-2-oxo-1-(2-trimethylsilanylethoxymethyl)-1,2-dihydrospiro[indole-

3,3'-pyrrolidine]-1'-carboxylic acid tert-butyl ester 13 and

5-Methoxy-2-oxo-1-(2-trimethylsilylethoxymethyl)-1,2-dihydro-spiro[indole-3,3'-

pyrrolidine]-1'-carboxylic acid tert-butyl ester 14b

5-Methoxy-2-oxo-1,2-dihydrospiro[ondole-3,3'-pyrrolidine]-1'-carboxylic acid

tert-butyl ester

Horsfiline 1

8

Pyrrolidine-1,3-dicarboxylic acid 1-benzyl ester 3-methyl ester 10b

9

3-Phenylselanylpyrrolidine-1,3-dicarboxylic acid 1-benzyl ester 3-methyl ester 15

9

3-(4-Methoxyphenylcarbamoyl)-3-phenylselanylpyrrolidine-1-carboxylic acid benzyl ester 16

10

3-[Benzyl-(4-methoxyphenyl)carbamoyl]-3-phenylselanylpyrrolidine-1-carboxylic acid benzyl ester 17

1-Benzyl-5-methoxy-2-oxo-1,2-dihydro-spiro[indole-3,3'-pyrrolidine]-1'- carboxylic acid benzyl ester 19 
All reagents were obtained from commercial suppliers unless otherwise stated. Where necessary, organic solvents were routinely dried and/or distilled prior to use and stored over molecular sieves under nitrogen. Diethyl ether was dried over sodium wire. Other solvents were dried by distillation from the following: tetrahydrofuran (sodium/benzophenone); dichloromethane (calcium hydride); ethyl acetate (magnesium sulfate). All reactions requiring anhydrous conditions were performed in flamedried apparatus under a nitrogen atmosphere. Organic extracts were, in general, dried over anhydrous magnesium sulfate $\left(\mathrm{MgSO}_{4}\right)$ or sodium sulfate $\left(\mathrm{Na}_{2} \mathrm{SO}_{4}\right)$.

Preparation of Benzyl 1-tert-\{butyloxycarbonyl\}pyrrolidine-3-carboxylate 10a:

stage 1: 1-tert-\{(Butyloxy)carbonyl\}-3-(methyloxycarbonyl)pyrrolidine:

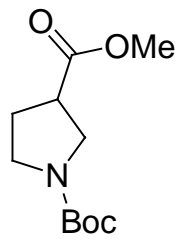

1-Benzylpyrrolidine-3-carboxylic acid methyl ester (1.13 g, $5.15 \mathrm{mmol}, 1$ equiv.) was dissolved in ethyl acetate $(100 \mathrm{ml})$. Then palladium on carbon $(10 \%, 385 \mathrm{mg})$ was added followed by di-tert-butyl dicarbonate (1.35 g, $6.18 \mathrm{mmol}, 1.2$ equiv). Hydrogenation was carried out at room temperature for $8 \mathrm{~h}$ and the suspension was then filtered through celite and the filtrate was concentrated under vacuum. The residue was purified by flash chromatography on silica gel (75:25, hexanes-ethyl acetate) giving 1-tert\{butyloxycarbonyl\}-3-(methyloxycarbonyl)pyrrolidine as a pale oil $(1.06 \mathrm{~g}, 90 \%) ; v_{\max }(\mathrm{film}) / \mathrm{cm}^{-1}$ 2977, 2883, $1742(\mathrm{C}=\mathrm{O}), 1702(\mathrm{C}=\mathrm{O}), 1411,1173,1129,882 ; \delta_{\mathrm{H}}\left(400 \mathrm{MHz}, \mathrm{CDCl}_{3}\right) 1.21\left(9 \mathrm{H}, \mathrm{s},{ }^{\mathrm{t}} \mathrm{Bu}\right)$, 1.85-1.90 (2H, m, CH$), 2.77-2.83\left(1 \mathrm{H}, \mathrm{m}, \mathrm{CH}_{2}\right), 3.07-3.13\left(1 \mathrm{H}, \mathrm{m}, \mathrm{CH}_{2}\right), 3.19-3.32\left(2 \mathrm{H}, \mathrm{m}, \mathrm{CH}_{2}\right)$, 3.35-3.37 (1H, m, CH), $3.46\left(3 \mathrm{H}, \mathrm{s}, \mathrm{CH}_{3}\right) ; \delta_{\mathrm{C}}\left(100.61 \mathrm{MHz}, \mathrm{CDCl}_{3}\right) 28.4\left(\mathrm{CH}_{3}\right), 29.5\left(\mathrm{CH}_{2}\right), 42.8(\mathrm{CH})$, $45.1\left(\mathrm{CH}_{2}\right), 48.1\left(\mathrm{CH}_{2}\right), 51.7\left(\mathrm{CH}_{3}\right), 79.1(\mathrm{C}), 154.1(\mathrm{C}), 173.2(\mathrm{C}) ; \mathrm{m} / \mathrm{Z}\left(\mathrm{EI}^{+}\right) 229\left[\left(\mathrm{M}^{+}\right), 29 \%\right], 172$ (100), 156 (74), $142(26), 128$ (36), $101(6), 82(5)$ and 57 (68).

stage 2: Pyrrolidine-1,3-dicarboxylic acid 1-tert-butyl ester:

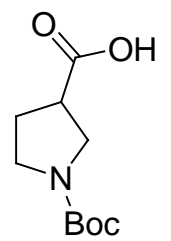

1-tert-\{(Butyloxy)carbonyl\}-3-(methyloxycarbonyl)pyrrolidine (2.23 g, $9.73 \mathrm{mmol}$, 1 equiv.) was dissolved in methanol $(40 \mathrm{ml})$. Then sodium hydroxide in solution in water $(0.78 \mathrm{~g}, 19.46 \mathrm{mmol}, 2$ equiv.) was added. The mixture was stirred at room temperature for $2 \mathrm{~h}$. The solvent was removed in vacuo. The remaining solution was acidified with acetic acid (2M) to $\mathrm{pH} 4$ and extracted with dichloromethane. The combined organic layers were dried over sodium sulfate and evaporated under reduced pressure to give the pyrrolidine-1,3-dicarboxylic acid 1-tert-butyl ester as a white solid (1.99 g, 95\%); mp $136-137^{\circ} \mathrm{C}$ [Found (ESI): $\left[\mathrm{MH}^{+}\right]$216.1233. $\mathrm{C}_{10} \mathrm{H}_{17} \mathrm{NO}_{4}$ requires $\mathrm{MH}$ 216.1236]; $v_{\max }$ 
(film) $/ \mathrm{cm}^{-1} 3511,3016,2981,2885,1717(\mathrm{C}=\mathrm{O}), 1693(\mathrm{C}=\mathrm{O}), 1419,1168 ; \delta_{\mathrm{H}}\left(400 \mathrm{MHz}, \mathrm{CDCl}_{3}\right) 1.25$ $(1 \mathrm{H}, \mathrm{s}, \mathrm{OH}), 1.47\left(9 \mathrm{H}, \mathrm{s},{ }^{\mathrm{t}} \mathrm{Bu}\right), 2.16-2.20\left(2 \mathrm{H}, \mathrm{m}, \mathrm{CH}_{2}\right), 3.08-3.12\left(1 \mathrm{H}, \mathrm{m}, \mathrm{CH}_{2}\right), 3.36-3.41(1 \mathrm{H}, \mathrm{m}$, $\left.\mathrm{CH}_{2}\right), 3.56-3.74\left(3 \mathrm{H}, \mathrm{m}, \mathrm{CH}_{2}\right.$ and $\left.\mathrm{CH}\right) ; \delta_{\mathrm{C}}\left(100.61 \mathrm{MHz}, \mathrm{CDCl}_{3}\right) 28.6\left(\mathrm{CH}_{3}\right), 29.5\left(\mathrm{CH}_{2}\right), 42.8(\mathrm{CH})$, $45.5\left(\mathrm{CH}_{2}\right), 48.2\left(\mathrm{CH}_{2}\right), 80.1(\mathrm{C}), 154.7(\mathrm{C}), 177.8(\mathrm{C}) ; \mathrm{m} / \mathrm{z}\left(\mathrm{EI}^{+}\right) 215$ [(M+), 2\%], 199 (1), 160 (14), 142 (32), 57 (100) and 41 (63).

\section{Stage 3: Benzyl 1-tert-\{butyloxycarbonyl\}pyrrolidine-3-carboxylate 10a:}

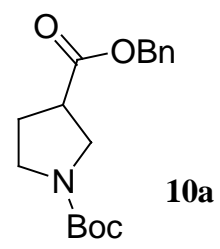

Pyrrolidine-1,3-dicarboxylic acid 1-tert-butyl ester (2.89 g, $13.43 \mathrm{mmol}, 1$ equiv.) was dissolved in benzyl bromide $(2.40 \mathrm{ml}, 20.15 \mathrm{mmol}, 1.5$ equiv.). Then 1,8-diazabicyclo[5.4.0]undec-7-ene (3.07 $\mathrm{g}$, 20.15 mmol, 1.5 equiv.) in benzene $(20 \mathrm{ml})$ was added under a nitrogen atmosphere. The solution was stirred at room temperature for $12 \mathrm{~h}$. The solution was washed with water $(3 \mathrm{x} 30 \mathrm{ml})$, then extracted with dichloromethane $(4 \times 30 \mathrm{ml})$. The combined organic layers were dried over sodium sulfate before being concentrated under vacuum. The residue was purified by column chromatography (75:25, hexanes-ethyl acetate) to give the benzyl 1-tert-\{butyloxycarbonyl\}pyrrolidine-3-carboxylate 10a as a pale oil (3.86 g, 94\%); (Found: C, 66.90; H, 7.82; N, 4.50. $\mathrm{C}_{17} \mathrm{H}_{23} \mathrm{NO}_{4}$ requires C, 66.85; H, 7.6; N, 4.59\%) [Found (ESI): $\mathrm{MH}^{+}$306.1707. $\mathrm{C}_{17} \mathrm{H}_{23} \mathrm{NO}_{4}$ requires $\mathrm{MH} 306.1705$ ]; $v_{\max }$ (film)/cm $\mathrm{cm}^{-1} 3061,3035$, 2972, 2889, $1741(\mathrm{C}=\mathrm{O}), 1703(\mathrm{C}=\mathrm{O}), 1468,1404,1170,1119 ; \delta_{\mathrm{H}}\left(400 \mathrm{MHz}, \mathrm{CDCl}_{3}\right) 1.46\left(9 \mathrm{H}, \mathrm{s},{ }^{\mathrm{t}} \mathrm{Bu}\right)$, 2.12-2.16 (2H, m, $\left.\mathrm{CH}_{2}\right), 3.07-3.10\left(1 \mathrm{H}, \mathrm{m}, \mathrm{CH}_{2}\right), 3.35-3.60\left(4 \mathrm{H}, \mathrm{m}, \mathrm{CH}_{2}\right.$ and $\left.\mathrm{CH}\right), 5.15\left(2 \mathrm{H}, \mathrm{s}, \mathrm{CH}_{2}\right)$, $7.34(5 \mathrm{H}, \mathrm{m}, \mathrm{ArH}) ; \delta_{\mathrm{C}}\left(100.61 \mathrm{MHz}, \mathrm{CDCl}_{3}\right) 28.8\left(\mathrm{CH}_{3}\right), 29.5\left(\mathrm{CH}_{2}\right), 42.8(\mathrm{CH}), 45.5\left(\mathrm{CH}_{2}\right), 48.4$ $\left(\mathrm{CH}_{2}\right), 66.8\left(\mathrm{CH}_{2}\right), 79.6(\mathrm{C}), 128.3(\mathrm{CH}), 128.5(\mathrm{CH}), 128.8(\mathrm{CH}), 136.2(\mathrm{C}), 154.6(\mathrm{C}), 173.1(\mathrm{C}) ; \mathrm{m} / \mathrm{z}$ $\left(\mathrm{CI}^{+}\right) 323\left[\left(\mathrm{MNH}_{4}^{+}\right), 100 \%\right], 306\left[\left(\mathrm{MH}^{+}\right), 48\right], 302$ (79), $282(68)$ and 267 (67).

\section{N-2-Iodo-4-methoxyphenyl-1-tert-\{butyloxycarbonyl\}pyrrolidine-3-carboxamide 12a:}

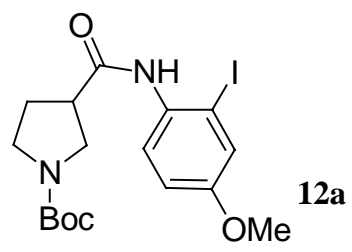

A solution of triethylaluminum in toluene $(1.9 \mathrm{M}, 2.54 \mathrm{ml}, 4.83 \mathrm{mmol}, 2.5$ equiv.) was added to a solution of 4-amino-3-iodoanisole $11\left(0.48 \mathrm{~g}, 1.93 \mathrm{mmol}, 1\right.$ equiv.) in toluene $(5 \mathrm{ml})$ at $0^{\circ} \mathrm{C}$ under inert atmosphere. The mixture was then warmed to room temperature. After stirring for $10 \mathrm{~min}$, a solution of benzyl 1-tert-\{butyloxycarbonyl\}pyrrolidine-3-carboxylate $\mathbf{1 0 a}(0.59 \mathrm{~g}, 1.93 \mathrm{mmol}$, 1 equiv.) in toluene $(5 \mathrm{ml})$ was added, and the mixture was heated at $80^{\circ} \mathrm{C}$ for $12 \mathrm{~h}$. On completion of the reaction, a saturated aqueous solution of ammonium chloride and a few drops of hydrochloric acid (2M) were added. The mixture was washed with a solution of sodium hydroxide (2M), and extracted with diethyl ether, and the combined organic phase was dried over sodium sulfate. The product was purified by 
column chromatography (70:30, hexanes-ethyl acetate) giving N-2-iodo-4-methoxyphenyl-1-tert\{butyloxycarbonyl \}pyrrolidine-3-carboxamide 12a as a white powder $(0.58 \mathrm{~g}, 66 \%)$; mp $147-148^{\circ} \mathrm{C}$ (Found: C, 45.84; H, 5.11; N, 6.28. $\mathrm{C}_{17} \mathrm{H}_{23} \mathrm{IN}_{2} \mathrm{O}_{4}$ requires $\mathrm{C}, 45.73 ; \mathrm{H}, 5.20 ; \mathrm{N}, 6.28 \%$ ) (Found (ESI): $\left[\mathrm{MH}^{+}\right]$447.0769. $\mathrm{C}_{17} \mathrm{H}_{23} \mathrm{IN}_{2} \mathrm{O}_{4}$ requires $\left.\mathrm{MH} 447.0781\right) ; v_{\max }($ film $) / \mathrm{cm}^{-1} 3251,2979,2938,1699(\mathrm{C}=\mathrm{O})$, $1670(\mathrm{C}=\mathrm{O}), 1415,1134 ; \delta_{\mathrm{H}}\left(400 \mathrm{MHz}, \mathrm{CDCl}_{3}\right) 1.48\left(9 \mathrm{H}, \mathrm{s},{ }^{\mathrm{t}} \mathrm{Bu}\right), 2.18-2.22\left(2 \mathrm{H}, \mathrm{m}, \mathrm{CH}_{2}\right), 3.05-3.09$ $\left(1 \mathrm{H}, \mathrm{m}, \mathrm{CH}_{2}\right), 3.36-3.48\left(1 \mathrm{H}, \mathrm{m}, \mathrm{CH}_{2}\right), 3.58-3.65\left(3 \mathrm{H}, \mathrm{m}, \mathrm{CH}\right.$ and $\left.\mathrm{CH}_{2}\right), 3.79(3 \mathrm{H}, \mathrm{s}, \mathrm{OMe}), 6.90(1 \mathrm{H}$, $\mathrm{dd}, J$ 8.8, 2.7, ArH), $7.31(1 \mathrm{H}, \mathrm{d}, J 2.7, \mathrm{ArH}), 7.37(1 \mathrm{H}, \mathrm{s}, \mathrm{NH}), 7.91(1 \mathrm{H}, \mathrm{d}, J 8.8, \operatorname{ArH}) ; \delta_{\mathrm{C}}(100.61$ $\left.\mathrm{MHz}, \mathrm{CDCl}_{3}\right) 28.9\left(\mathrm{CH}_{3}\right), 29.6\left(\mathrm{CH}_{2}\right), 45.8\left(\mathrm{CH}_{2}\right), 46.2(\mathrm{CH}), 48.9\left(\mathrm{CH}_{2}\right), 56.2\left(\mathrm{CH}_{3}\right), 80.0(\mathrm{C}), 92.6$ (C), $115.2(\mathrm{CH}), 124.2(\mathrm{CH}), 124.6(\mathrm{CH}), 131.7(\mathrm{C}), 154.8(\mathrm{C}), 157.5(\mathrm{C}), 171.1(\mathrm{C}) ; \mathrm{m} / z\left(\mathrm{CI}^{+}\right) 447$ $\left[\left(\mathrm{M}^{+}\right), 100 \%\right], 418(9)$ and $402(17)$.

\section{$N$-(Trimethylsilyl)ethoxymethyl- $N$-[2-iodo-4-methoxyphenyl]-1-tert-\{butyloxycarbonyl\}- pyrrolidine-3-carboxamide 12b:}

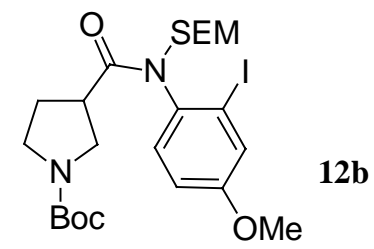

$\mathrm{N}$-2-Iodo-4-methoxyphenyl-1-tert-\{butyloxycarbonyl\}pyrrolidine-3-carboxamide 12a (0.58 g, 1.30 mmol, 1 equiv.) in dry tetrahydrofuran $(20 \mathrm{ml})$ was added to a stirred solution of sodium hydride $(60 \%$ in mineral oil, $0.057 \mathrm{~g}, 1.43 \mathrm{mmol}, 1.1$ equiv.) (washed twice with dry THF) in dry tetrahydrofuran (30 $\mathrm{ml})$. When hydrogen evolution ceased, 2-(trimethylsilyl)ethoxymethyl chloride $(0.23 \mathrm{ml}, 1.30 \mathrm{mmol}, 1$ equiv.) was added dropwise at $0^{\circ} \mathrm{C}$. The reaction mixture was left to come to room temperature and stirred for $3 \mathrm{~h}$. On completion, water $(20 \mathrm{ml})$ was added to destroy the remaining sodium hydride. Extraction was performed using dichloromethane $(3 \times 30 \mathrm{ml})$. The organic phase was dried over sodium sulfate, and solvent was evaporated under vacuum. The crude product was purified by column chromatography (70:30, hexanes-ethyl acetate) giving $N$-(trimethylsilyl)ethoxymethyl- $N$-[2-iodo-4methoxyphenyl]-1-tert-\{butyloxycarbonyl \}pyrrolidine-3-carboxamide $\mathbf{1 2 b}(0.75 \mathrm{~g}, 100 \%)$ as a pale oil; (Found (ESI): $\left[\mathrm{MH}^{+}\right]$577.1594. $\mathrm{C}_{23} \mathrm{H}_{37} \mathrm{IN}_{2} \mathrm{O}_{5}$ Si requires $\mathrm{MH}$ 577.1595); $v_{\max }(\mathrm{film}) / \mathrm{cm}^{-1} 3053,2979$, 2892, $1678(\mathrm{C}=\mathrm{O}), 1677(\mathrm{C}=\mathrm{O}), 1593,1490,1402 ; \delta_{\mathrm{H}}\left(400 \mathrm{MHz}, \mathrm{CDCl}_{3}\right) 0.06\left(9 \mathrm{H}, \mathrm{s}, \mathrm{SiMe}_{3}\right), 0.81-$ $0.96\left(2 \mathrm{H}, \mathrm{m}, \mathrm{CH}_{2}-\mathrm{Si}\right), 1.36\left(9 \mathrm{H}, \mathrm{s},{ }^{\mathrm{t}} \mathrm{Bu}\right), 1.87-1.96\left(1 \mathrm{H}, \mathrm{m}, \mathrm{CH}_{2}\right), 2.63\left(1 \mathrm{H}, \mathrm{m}, \mathrm{CH}_{2}\right), 3.03-3.09(1 \mathrm{H}, \mathrm{m}$, $\left.\mathrm{CH}_{2}\right), 3.21-3.29\left(1 \mathrm{H}, \mathrm{m}, \mathrm{CH}_{2}\right), 3.45-3.75\left(5 \mathrm{H}, \mathrm{m}, \mathrm{CH}\right.$ and $\left.\mathrm{CH}_{2}\right), 4.05(3 \mathrm{H}, \mathrm{s}, \mathrm{OMe}), 4.24(1 \mathrm{H}, \mathrm{d}, J 10$, $\left.\mathrm{NCH}_{2} \mathrm{O}\right), 5.57\left(1 \mathrm{H}, \mathrm{d}, J 10, \mathrm{NCH}_{2} \mathrm{O}\right), 6.87-6.89(1 \mathrm{H}, \mathrm{m}, \mathrm{ArH}), 7.06-7.11(1 \mathrm{H}, \mathrm{m}, \mathrm{ArH}), 7.39(1 \mathrm{H}, \mathrm{m}$, $\mathrm{ArH}) ; \delta_{\mathrm{C}}\left(100.61 \mathrm{MHz}, \mathrm{CDCl}_{3}\right)-1.4\left(\mathrm{CH}_{3}\right), 17.9\left(\mathrm{CH}_{2}\right), 28.3\left(\mathrm{CH}_{3}\right), 28.8\left(\mathrm{CH}_{2}\right), 42.0(\mathrm{CH}), 45.4\left(\mathrm{CH}_{2}\right)$, $49.1\left(\mathrm{CH}_{2}\right), 55.5\left(\mathrm{CH}_{3}\right), 66.1\left(\mathrm{CH}_{2}\right), 76.3\left(\mathrm{CH}_{2}\right), 78.9(\mathrm{C}), 101.0(\mathrm{C}), 114.9(\mathrm{CH}), 124.8(\mathrm{CH}), 130.9$ $(\mathrm{CH}), 135.4(\mathrm{C}), 153.9(\mathrm{C}), 159.5$ (C), 173.6 (C); m/z (ESI) 577 [( $\left.\left.\mathrm{MH}^{+}\right), 100 \%\right], 521$ (10), 334 (11) and 79 (6). 
5-Methoxy-2-oxo-1-(2-trimethylsilanylethoxymethyl)-1,2-dihydrospiro[indole-3,3'-pyrrolidine]1 '-carboxylic acid tert-butyl ester 13 and 5-methoxy- $N$-(trimethylsilyl)ethoxymethyl- $N$-phenyl-1tert-\{butyloxycarbonyl\}-pyrrolidine-3-carboxamide 14a

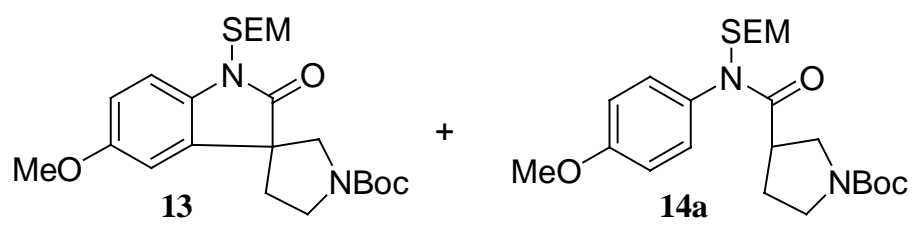

$N$-(Trimethylsilyl)ethoxymethyl- $N$-[2-iodo-4-methoxyphenyl]-1-tert- $\{$ butyloxycarbonyl $\}$ pyrrolidine-3carboxamide 12b (1.42 g, $2.46 \mathrm{mmol}, 1$ equiv.) was dissolved in refluxing dry toluene (10 ml), under nitrogen atmosphere. Using a syringe pump (minimum possible flow), 1-ethylpiperidine hypophosphite (4.40 g, $24.6 \mathrm{mmol}, 10$ equiv.) and azobisisobutyronitrile ( $0.40 \mathrm{~g}, 2.46 \mathrm{mmol}, 1$ equiv.) in solution in 4 $\mathrm{ml}$ of toluene, were added under nitrogen atmosphere overnight. On completion, water. was added. Toluene was evaporated, the crude product was diluted in dichloromethane, washed with hydrochloric acid $(2 \mathrm{M}, 30 \mathrm{ml})$, sodium hydrogencarbonate $(2 \mathrm{M}, 30 \mathrm{ml})$, brine, the organic phase was dried over sodium sulfate, and evaporated under reduced pressure. The crude product was purified by column chromatography $(80: 20$, hexanes-ethyl acetate) to give 5-methoxy-2-oxo-1-(2trimethylsilanylethoxymethyl)-1,2-dihydrospiro[indole-3,3'-pyrrolidine]-1'-carboxylic acid tert-butyl ester 13 as a pale oil $(0.368 \mathrm{~g}, 33 \%)$ and 5-methoxy- $N$-(trimethylsilyl)ethoxymethyl- $N$-phenyl-1-tert\{butyloxycarbonyl pyrrolidine-3-carboxamide $\mathbf{1 4 a}$ as a pale oil $(0.566 \mathrm{~g}, 51 \%)$.

5-Methoxy-2-oxo-1-(2-trimethylsilanylethoxymethyl)-1,2-dihydrospiro[indole-3,3'-pyrrolidine]-1'carboxylic acid tert-butyl ester 13: (Found $\left(\mathrm{EI}^{+}\right)$: $\left[\mathrm{M}^{+}\right]$448.2398. $\mathrm{C}_{23} \mathrm{H}_{3} \mathrm{~N}_{2} \mathrm{O}_{5} \mathrm{Si}$ requires $\mathrm{M}$ 448.2394); $\nu_{\max }\left(\right.$ film)/cm ${ }^{-1}$ 3006, 2954, 2894, $1721(\mathrm{C}=\mathrm{O}), 1699(\mathrm{C}=\mathrm{O}), 1496,1400 ; \delta_{\mathrm{H}}\left(400 \mathrm{MHz}, \mathrm{CDCl}_{3}\right) 0.03$ (9H, s, $\left.\mathrm{SiMe}_{3}\right), 0.89-0.93\left(2 \mathrm{H}, \mathrm{m}, \mathrm{CH}_{2}\right), 1.42(9 \mathrm{H}, 2$ x s, Boc), 2.02-2.05 (1H, m, CH$), 2.38-2.45(1 \mathrm{H}$, $\left.\mathrm{m}, \mathrm{CH}_{2}\right), 3.52-3.57\left(2 \mathrm{H}, \mathrm{m}, \mathrm{CH}_{2}\right), 3.62-3.86\left(4 \mathrm{H}, \mathrm{m}, \mathrm{CH}_{2}\right), 3.80(3 \mathrm{H}, \mathrm{s}, \mathrm{OMe}), 5.11-5.18\left(2 \mathrm{H}, \mathrm{m}, \mathrm{CH}_{2}\right)$, 6.82-6.84 (2H, m, ArH), 6.98-7.00 (1H, m, ArH); $\delta_{\mathrm{C}}\left(100.61 \mathrm{MHz}, \mathrm{CDCl}_{3}\right)-1.3\left(\mathrm{CH}_{3}\right), 17.9\left(\mathrm{CH}_{2}\right)$, $28.6\left(\mathrm{CH}_{3}\right), 35.9\left(\mathrm{CH}_{2}\right), 36.8(\mathrm{C}), 45.5\left(\mathrm{CH}_{2}\right), 54.8\left(\mathrm{CH}_{2}\right), 56.0\left(\mathrm{CH}_{3}\right), 66.1\left(\mathrm{CH}_{2}\right), 69.7\left(\mathrm{CH}_{2}\right), 79.9(\mathrm{C})$, $110.0(\mathrm{CH}), 110.4(\mathrm{CH}), 112.9(\mathrm{CH}), 133.7(\mathrm{C}), 134.7(\mathrm{C}), 154.5(\mathrm{C}), 156.8(\mathrm{C}), 177.8(\mathrm{C}) ; \mathrm{m} / z\left(\mathrm{EI}^{+}\right)$ $448\left[\left(\mathrm{M}^{+}\right), 100 \%\right], 433(1), 405$ (3), $389(9)$ and 375 (23).

5-methoxy- $N$-(trimethylsilyl)ethoxymethyl- $N$-phenyl-1-tert- $\{$ butyloxycarbonyl $\}$-pyrrolidine-3carboxamide 14a: (Found (ESI): $\left[\mathrm{MH}^{+}\right]$451.2624. $\mathrm{C}_{23} \mathrm{H}_{38} \mathrm{~N}_{2} \mathrm{O}_{5} \mathrm{Si}$ requires $\mathrm{MH}$ 451.2628); $v_{\max }$ (film)/cm ${ }^{-1} 2954,2894,1694(\mathrm{C}=\mathrm{O}), 1695(\mathrm{C}=\mathrm{O}), 1513,1455,1403 ; \delta_{\mathrm{H}}\left(400 \mathrm{MHz}, \mathrm{CDCl}_{3}\right) 0.03(9 \mathrm{H}, \mathrm{s}$, $\left.\mathrm{SiMe}_{3}\right), 0.93\left(2 \mathrm{H}, \mathrm{t}, J\right.$ 8.3, $\left.\mathrm{CH}_{2}\right), 1.41(9 \mathrm{H}, \mathrm{s}, \mathrm{Boc}), 1.84-1.86\left(1 \mathrm{H}, \mathrm{m}, \mathrm{CH}_{2}\right), 2.03\left(1 \mathrm{H}, \mathrm{m}, \mathrm{CH}_{2}\right), 2.80$ $\left(1 \mathrm{H}, \mathrm{m}, \mathrm{CH}_{2}\right), 3.10-3.12\left(1 \mathrm{H}, \mathrm{m}, \mathrm{CH}_{2}\right), 3.40-3.52\left(3 \mathrm{H}, \mathrm{m}, \mathrm{CH}\right.$ and $\left.\mathrm{CH}_{2}\right), 3.59-3.63-\left(2 \mathrm{H}, \mathrm{m}, \mathrm{CH}_{2}\right), 3.82$ (3H, s, OMe), 4.99-5.05 (2H, m, $\left.\mathrm{CH}_{2}\right), 6.92(2 \mathrm{H}, \mathrm{d}, J$ 8.4, $\left.\mathrm{ArH}), 7.08-7.12(2 \mathrm{H}, \mathrm{m}, \mathrm{ArH})\right) ; \delta_{\mathrm{C}}(100.61$ $\left.\mathrm{MHz}, \mathrm{CDCl}_{3}\right)$-1.2 $\left(\mathrm{CH}_{3}\right), 18.3\left(\mathrm{CH}_{2}\right), 28.7\left(\mathrm{CH}_{3}\right), 29.9\left(\mathrm{CH}_{2}\right), 42.0(\mathrm{CH}), 45.6\left(\mathrm{CH}_{2}\right), 45.8\left(\mathrm{CH}_{2}\right), 49.4$ $\left(\mathrm{CH}_{2}\right), 55.7\left(\mathrm{CH}_{3}\right), 66.2\left(\mathrm{CH}_{2}\right), 79.4(\mathrm{C}), 115.1(\mathrm{CH}), 129.7(\mathrm{CH}), 134.2(\mathrm{C}), 154.4(\mathrm{C}), 159.6(\mathrm{C})$, $174.0(\mathrm{C}) ; \mathrm{m} / z\left(\mathrm{CI}^{+}\right) 451$ [( $\left.\left.\mathrm{MH}^{+}\right), 48 \%\right], 351$ (32), 333 (43), 233 (100), 136 (38) and 90 (55). 
5-Methoxy-2-oxo-1-(2-trimethylsilanylethoxymethyl)-1,2-dihydrospiro[indole-3,3'-pyrrolidine]1 '-carboxylic acid tert-butyl ester 13 and 5-Methoxy-2-oxo-1-(2-trimethylsilanylethoxymethyl)1,2-dihydro-spiro[indole-3,3'-pyrrolidine]-1'-carboxylic acid tert-butyl ester 14b:

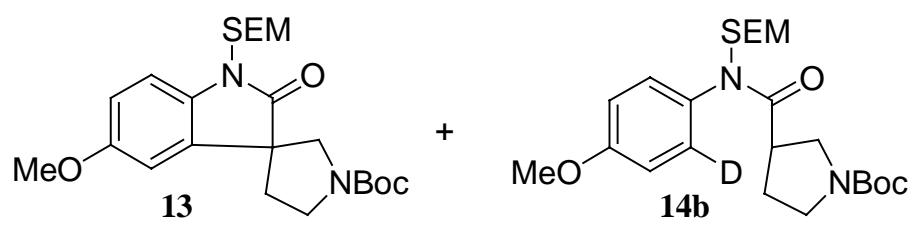

Deuterium oxide $\left(\mathrm{D}_{2} \mathrm{O}\right)$ was removed via Dean Stark-trap from the mixture of deuterated hypophosphorous acid (0.648 g, $9.38 \mathrm{mmol}, 10.0$ equiv., $50 \%$ in $\left.\mathrm{D}_{2} \mathrm{O}\right)$ and benzene $(50 \mathrm{ml})$ under nitrogen. Ethylpiperidine (1.23 g, $10 \mathrm{mmol}, 10$ equiv.) was then added to the reaction mixture. $\mathrm{N}$ (Trimethylsilyl)ethoxymethyl- $N$-(2-iodo-4-methoxyphenyl)-1-tert-(butyloxycarbonyl) pyrrolidine-3carboxamide 12b $(0.54 \mathrm{~g}, 0.938 \mathrm{mmol}, 1$ equiv.) was dissolved in benzene $(10 \mathrm{ml})$ and added to the reaction mixture. Finally azobisisobutyronitrile $(4 \mathrm{x} 0.057 \mathrm{~g}, 1.0 \mathrm{mmol}, 1.5$ equiv.) was added to the reaction mixture every $12 \mathrm{~h}$ under refluxed in nitrogen atmosphere. On completion, water (15 ml) was added. Benzene was evaporated in vacuo and the crude product was purified by column chromatography (80:20, ethyl acetate/hexanes) to afford desired cyclized product 5-methoxy-2-oxo-1(2-trimethylsilanylethoxymethyl)-1,2-dihydrospiro-[indole-3,3'-pyrrolidine]-1'-carboxylic acid tertbutyl ester 13 as a pale yellow oil (0.252 g, 60\%) along with reduced deuterated side product 3-[(4methoxy-phenyl)-(2-trimethylsilanyl-ethoxymethyl)-carbamoyl]-pyrrolidine-1-carboxylic acid tertbutyl ester 14b (0.153 g, 36\%); Found: (ESI) $\mathrm{MH}^{+}$, 452.2683. $\mathrm{C}_{23} \mathrm{H}_{37} \mathrm{DN}_{2} \mathrm{O}_{5} \mathrm{Si}$ requires $\mathrm{MH}, 452.2686$ ); $v_{\max }\left(\right.$ film) $/ \mathrm{cm}^{-1}$ 2952, 2893, 1697, 1670, 1496, 1399, 1248, 1171 and 1076; (2H NMR Spectrum acquired at $61 \mathrm{MHz}(1 \mathrm{H}=400 \mathrm{MHz}) 128$ transient. Calibrated against unlocked $\left.\mathrm{CDCl}_{3}\right) \delta_{\mathrm{D}} 7.15(1 \mathrm{D}, \mathrm{s}$, $\operatorname{ArD}) ; \delta_{\mathrm{H}}\left(400 \mathrm{MHz}, \mathrm{CDCl}_{3}\right) 0.00\left(9 \mathrm{H}, \mathrm{s}, \mathrm{SiCH}_{3}\right), 0.94\left(2 \mathrm{H}, \mathrm{t}, J\right.$ 8.4, $\left.\mathrm{CH}_{2}\right), 1.39\left(9 \mathrm{H}, \mathrm{s}, \mathrm{CH}_{3}\right), 1.73-1.77$ $\left(1 \mathrm{H}, \mathrm{m}, \mathrm{CH}_{2}\right), 2.13\left(1 \mathrm{H}, \mathrm{m}, \mathrm{CH}_{2}\right), 2.80\left(1 \mathrm{H}, \mathrm{m}, \mathrm{CH}_{2}\right), 3.11-3.13\left(1 \mathrm{H}, \mathrm{m}, \mathrm{CH}_{2}\right), 3.40-3.57(3 \mathrm{H}, \mathrm{m}, \mathrm{CH}$ and $\left.\mathrm{CH}_{2}\right), 3.59-3.64\left(2 \mathrm{H}, \mathrm{t}, J\right.$ 8.4, $\left.\mathrm{CH}_{2}\right), 3.83\left(3 \mathrm{H}, \mathrm{s}, \mathrm{OCH}_{3}\right), 5.00-5.05\left(2 \mathrm{H}, \mathrm{m}, \mathrm{CH}_{2}\right), 6.92(2 \mathrm{H}, \mathrm{m}$, ArH), $7.11(1 \mathrm{H}, \mathrm{m}, \mathrm{ArH}))$; $\delta_{\mathrm{C}}\left(100.61 \mathrm{MHz}, \mathrm{CDCl}_{3}\right)$-1.2 $\left(\mathrm{CH}_{3}\right), 18.3\left(\mathrm{CH}_{2}\right), 29.6\left(\mathrm{CH}_{3}\right), 29.8\left(\mathrm{CH}_{2}\right)$, $45.5(\mathrm{CH}), 45.7\left(\mathrm{CH}_{2}\right), 49.3\left(\mathrm{CH}_{2}\right), 55.7\left(\mathrm{CH}_{3}\right), 66.3\left(\mathrm{CH}_{2}\right), 76.9\left(\mathrm{CH}_{2}\right), 79.4(\mathrm{C}), 107.9(\mathrm{CH}), 115.1$ $(\mathrm{CH}), 129.6(\mathrm{CH}), 134.2(\mathrm{C}), 154.4(\mathrm{C}), 159.6(\mathrm{C})$ and $174.0(\mathrm{C}) ; \mathrm{m} / z(\mathrm{CI}) 452\left(\mathrm{M}+\mathrm{H}^{+}, 10 \%\right), 334(25)$, 234 (30), 137 (80) and 90 (100).

\section{Deprotection of 13: step 1}

5-Methoxy-2-oxo-1,2-dihydrospiro[indole-3,3'-pyrrolidine]-1'-carboxylic acid tert-butyl ester:

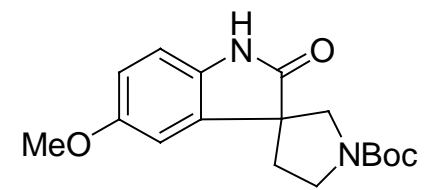

5-Methoxy-2-oxo-1-(2-trimethylsilanylethoxymethyl)-1,2-dihydrospiro[indole-3,3'-pyrrolidine]-1'carboxylic acid tert-butyl ester 13 (0.36 g, 0.82 mmol, 1 equiv.) was dissolved in dimethyl formamide (5 ml) and ethylenediamine $(2.2 \mathrm{ml})$ under nitrogen atmosphere. Then tetrabutylammonium fluoride-hydrate ( $0.65 \mathrm{~g}, 2.50 \mathrm{mmol}, 3.05$ equiv.) was added and the mixture heated at $150^{\circ} \mathrm{C}$ for $12 \mathrm{~h}$. On completion, 
water $(5 \mathrm{ml})$ was added and the reaction mixture extracted with diethyl ether. Organic extracts were then washed with hydrochloric acid $(2 \mathrm{~N})$ and saturated sodium hydrogencarbonate solution, before being dried over sodium sulfate and evaporated under reduced pressure. The residue was purified by column chromatography $(60: 40$, hexanes-ethyl acetate) to give 5-methoxy-2-oxo-1,2dihydrospiro[ondole-3,3'-pyrrolidine]-1'-carboxylic acid tert-butyl ester as a white foam (0.1232 g, 47\%); (Found: C, 64.17; H, 8.59; N, 6.76. $\mathrm{C}_{17} \mathrm{H}_{22} \mathrm{~N}_{2} \mathrm{O}_{4}$ requires C, 64.13; H, 8.80; N, 6.97\%) (Found (ESI): $\left[\mathrm{MH}^{+}\right]$319.1660. $\mathrm{C}_{17} \mathrm{H}_{22} \mathrm{~N}_{2} \mathrm{O}_{4}$ requires $\left.\mathrm{MH} 319.1658\right) ; v_{\max }(\mathrm{KBr}) / \mathrm{cm}^{-1} 3242(\mathrm{NH}), 2978,2892$, 1699, 1493, 1204, 1176, 1037; $\delta_{\mathrm{H}}\left(400 \mathrm{MHz}, \mathrm{CDCl}_{3}\right) 1.47\left(9 \mathrm{H}, 2\right.$ x s, Boc), 2.03-2.06 (1H, m, $\left.\mathrm{CH}_{2}\right)$, 2.36-2.43 (1H, m, $\left.\mathrm{CH}_{2}\right), 3.52-3.63\left(1 \mathrm{H}, \mathrm{m}, \mathrm{CH}_{2}\right), 3.76(3 \mathrm{H}, \mathrm{s}, \mathrm{OMe}), 3.70-3.85\left(3 \mathrm{H}, \mathrm{m}, \mathrm{CH}_{2}\right), 6.76(2 \mathrm{H}$, m, ArH), $6.85(1 \mathrm{H}, \mathrm{m}, \mathrm{ArH}), 9.40\left(1 \mathrm{H}, 2\right.$ x s, CONH); $\delta_{\mathrm{C}}\left(100.61 \mathrm{MHz}, \mathrm{CDCl}_{3}\right) 28.6\left(\mathrm{CH}_{3}\right), 35.6$ $\left(\mathrm{CH}_{2}\right), 36.5(\mathrm{C}), 45.5\left(\mathrm{CH}_{2}\right), 54.0\left(\mathrm{CH}_{2}\right), 55.9\left(\mathrm{CH}_{3}\right), 80.0(\mathrm{C}), 110.1(\mathrm{CH}), 110.5(\mathrm{CH}), 112.9(\mathrm{CH})$, 133.8 (C), $134.6(\mathrm{C}), 154.6(\mathrm{C}), 156.3(\mathrm{C}), 180.3(\mathrm{C}) ; \mathrm{m} / z\left(\mathrm{EI}^{+}\right) 318$ [(M+), 100\%].

\section{Horsfiline 1:}

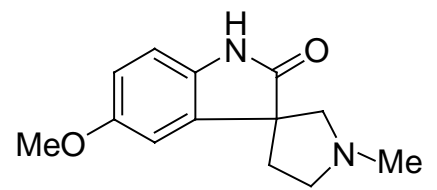

Trifluoroacetic acid $(0.29 \mathrm{ml}, 3.87 \mathrm{mmol}, 10$ equiv.) was added to a solution of 5-methoxy-2-oxo-1,2dihydrospiro[ondole-3,3'-pyrrolidine]-1'-carboxylic acid tert-butyl ester $(0.12 \mathrm{~g}, 0.38 \mathrm{mmol}, 1$ equiv.) in dry dichloromethane $(10 \mathrm{ml})$. The reaction was stirred at room temperature. On completion, water (10 $\mathrm{ml})$ and dichloromethane $(10 \mathrm{ml})$ were added. The layers were separated, and the aqueous layer was basified with sodium hydroxide solution $(2 \mathrm{~N})$ to $\mathrm{pH} 12$. Then it was extracted with dichloromethane, before being dried over sodium sulfate and evaporated under reduced pressure. The residue was not purified any further and was used crude to perform the following step.

The residue (crude amine) (0.387 mmol, 1 equiv.) was dissolved in dry dichloromethane (10 $\mathrm{ml})$ and methanol $(1.5 \mathrm{ml})$ under a nitrogen atmosphere. Formaldehyde $(1.3 \mathrm{ml})$ and $\mathrm{NaCNBH}_{3}(200 \mathrm{mg})$ were added. $\mathrm{pH}$ was adjusted to 3 with hydrochloric acid in methanol (10\%). After 5 min stirring, another portion of $\mathrm{NaCNBH}_{3}(200 \mathrm{mg})$ as added and the mixture was left to react overnight. On completion, water and dichloromethane were added (10 ml of each). Aqueous layer was then basified to $\mathrm{pH} 12$ before being extracted with dichloromethane, dried over sodium sulfate and evaporated under reduced pressure. The residue was purified by column chromatography (75:25, hexanes-ethyl acetate) to give horsfiline 1 as a white solid $\left(0.33 \mathrm{~g}, 89 \%\right.$ over 2 steps); mp $155-156^{\circ} \mathrm{C}$ [Found (ESI): [MH $\left.{ }^{+}\right] 233.1289$.

$\mathrm{C}_{13} \mathrm{H}_{16} \mathrm{~N}_{2} \mathrm{O}_{2}$ requires $\left.\mathrm{MH} 233.1290\right]$; $v_{\max }(\mathrm{KBr}) / \mathrm{cm}^{-1} 3400,2945,2836,1713(\mathrm{C}=\mathrm{O}), 1488,1209,1032$; $\delta_{\mathrm{H}}\left(400 \mathrm{MHz}, \mathrm{CDCl}_{3}\right)$ 2.06-2.13 $\left(1 \mathrm{H}, \mathrm{m}, \mathrm{CH}_{2}\right), 2.38-2.46\left(1 \mathrm{H}, \mathrm{m}, \mathrm{CH}_{2}\right), 2.47\left(3 \mathrm{H}, \mathrm{s}, \mathrm{NCH}_{3}\right), 2.72-2.78$ $\left(1 \mathrm{H}, \mathrm{m}, \mathrm{CH}_{2}\right), 2.84\left(1 \mathrm{H}, \mathrm{d}, J 9.4, \mathrm{CH}_{2}\right), 2.88\left(1 \mathrm{H}, \mathrm{d}, J\right.$ 9.4, $\left.\mathrm{CH}_{2}\right), 3.01-3.06\left(1 \mathrm{H}, \mathrm{m}, \mathrm{CH}_{2}\right), 3.81(3 \mathrm{H}, \mathrm{s}$, $\left.\mathrm{OCH}_{3}\right), 6.73(1 \mathrm{H}, \mathrm{dd}, J$ 8.4, 2.4, ArH), $6.77(1 \mathrm{H}, \mathrm{d}, J$ 8.4, $\mathrm{ArH}), 7.04(1 \mathrm{H}, \mathrm{d}, J 2.4, \mathrm{ArH}), 7.61(1 \mathrm{H}, \mathrm{bs}$, $\mathrm{NH}) ; \delta_{\mathrm{C}}\left(100.61 \mathrm{MHz}, \mathrm{CDCl}_{3}\right) 38.4\left(\mathrm{CH}_{2}\right), 42.0\left(\mathrm{CH}_{3}\right), 54.3(\mathrm{C}), 56.1\left(\mathrm{CH}_{3}\right), 56.9\left(\mathrm{CH}_{2}\right), 66.6\left(\mathrm{CH}_{2}\right)$, $109.8(\mathrm{CH}), 110.7(\mathrm{CH}), 112.7(\mathrm{CH}), 133.4(\mathrm{C}), 137.9(\mathrm{C}), 156.5(\mathrm{C}), 182.4(\mathrm{C}) ; \mathrm{m} / z\left(\mathrm{CI}^{+}\right) 233\left[\left(\mathrm{MH}^{+}\right)\right.$, $100 \%]$. 
Pyrrolidine-1,3-dicarboxylic acid 1-benzyl ester 3-methyl ester 10b:

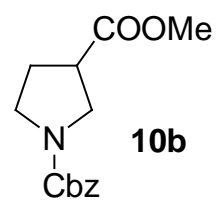

A solution of methyl acrylate $(1.72 \mathrm{~g}, 20.0 \mathrm{mmol}, 1.0$ equiv.) in benzene $(250 \mathrm{ml})$ was refluxed in a flask fitted with reflux condenser and Dean-Stark trap. To it a mixture of paraformaldehyde $(2.40 \mathrm{~g}$, $80.0 \mathrm{mmol}, 4.0$ equiv.) and glycine (3.0 g, $40 \mathrm{mmol}, 2.0$ equiv.) was added in portions over a period of $2 \mathrm{~h}$. The reaction mixture was refluxed for an additional $3 \mathrm{~h}$. It was then cooled, filtered and concentrated in vacuo to give pyrrolidine-3-carboxylic acid methyl ester, as a viscous liquid (2.1 g, $85 \%)$. The crude product was directly utilised for the next reaction without further purification. An aqueous solution $(20 \mathrm{ml})$ of sodium bicarbonate $(1.3 \mathrm{~g}, 15.5 \mathrm{mmol}, 2.0$ equiv.) was added to a toluene solution $(20 \mathrm{ml})$ of pyrrolidine-3-carboxylic acid methyl ester (1.0 g, $7.74 \mathrm{mmol}, 1.0 \mathrm{equiv}$.). The mixture was stirred vigorously and then benzyl chloroformate (1.72 g, $10.0 \mathrm{mmol}, 1.3$ equiv) was added dropwise at rt. The reaction mixture was left to stir overnight at room temperature, after which the organic fraction was separated. The aqueous fraction was extracted with ethyl acetate. The combined organic fractions were washed with aqueous hydrochloric acid solution (2N, $50 \mathrm{ml}$ ), followed by brine $(50 \mathrm{ml})$, dried over anhydrous sodium sulfate and concentrated in vacuo. Column chromatography (90:10, hexanes-ethyl acetate) afforded pyrrolidine-1,3-dicarboxylic acid 1-benzyl ester 3-methyl ester 10b as a yellow oil $(1.88 \mathrm{~g}, 94 \%)$. The product was found to be a mixture of two rotamers as observed from the ${ }^{13} \mathrm{C}$-NMR spectrometry (Found; $\left[\mathrm{M}+\mathrm{NH}_{4}\right]^{+}, 281.1498, \mathrm{C}_{14} \mathrm{H}_{17} \mathrm{NO}_{4}$ requires $\left.\left[\mathrm{M}+\mathrm{NH}_{4}\right]^{+} 281.1496\right) \cdot v_{\max }\left(\mathrm{film}, \mathrm{cm}^{-1}\right) 2954,1732,1713,1698,1498,1417,1361,1207$ and 1118. $\delta_{\mathrm{H}}\left(400 \mathrm{MHz} ; \mathrm{CDCl}_{3}\right) 2.13-2.17\left(2 \mathrm{H}, \mathrm{m}, \mathrm{CH}_{2}\right), 3.04-3.11(1 \mathrm{H}, \mathrm{m}, \mathrm{CH}), 3.39-3.68\left(4 \mathrm{H}, \mathrm{m}, \mathrm{CH}_{2}\right)$, $3.70\left(3 \mathrm{H}, \mathrm{s}, \mathrm{OCH}_{3}\right), 5.13\left(2 \mathrm{H}, \mathrm{s}, \mathrm{CH}_{2}\right), 7.28-7.38(5 \mathrm{H}, \mathrm{m}, \mathrm{ArCH}) ; \delta_{\mathrm{C}}\left(100.61 \mathrm{MHz}, \mathrm{CDCl}_{3}\right) 28.1$ and $28.8\left(\mathrm{CH}_{2}\right), 42.2$ and $43.0(\mathrm{CH}), 45.1$ and $45.6\left(\mathrm{CH}_{2}\right), 47.8$ and $48.4\left(\mathrm{CH}_{2}\right), 52.0\left(\mathrm{OCH}_{3}\right), 66.7\left(\mathrm{CH}_{2}\right)$, $127.8(\mathrm{CH}), 127.9(\mathrm{CH}), 128.4(\mathrm{CH}), 136.8(\mathrm{C}), 154.5(\mathrm{C}) 173.1$ and $173.3(\mathrm{C}) ; \mathrm{m} / \mathrm{z}\left(\mathrm{CI}^{+}\right) 281\left(\mathrm{MNH}_{4}^{+}\right)$ (100\%), 264 (50), $220(15), 130$ (15) and $108(10)$.

\section{3-Phenylselanylpyrrolidine-1,3-dicarboxylic acid 1-benzyl ester 3-methyl ester 15:}

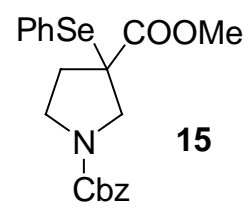

Diisopropylamine $(4.6 \mathrm{ml}, 32 \mathrm{mmol}, 1.5$ equiv.) was dissolved in dry THF (50 ml) and cooled to -78 ${ }^{\circ} \mathrm{C}$. To it $n$-BuLi (2.5 M in hexanes, $10.5 \mathrm{ml}, 26.0 \mathrm{mmol}, 1.2$ equiv.) was added dropwise under nitrogen and the mixture stirred for $1 \mathrm{~h}$. A solution of pyrrolidine-1,3-dicarboxylic acid 1-benzyl ester 3-methyl ester (5.76 g, $22.0 \mathrm{mmol}, 1.0$ equiv.) in dry THF $(50 \mathrm{ml})$ was added to the above mixture under nitrogen at $-78^{\circ} \mathrm{C}$ and stirred for $1 \mathrm{~h}$. A solution of phenylselenyl chloride $(5.0 \mathrm{~g}, 26.0 \mathrm{mmol}, 1.2$ equiv.) in dry THF $(50 \mathrm{ml})$ was then added to the above enolate solution at $-78{ }^{\circ} \mathrm{C}$ and the mixture stirred for $2 \mathrm{~h}$ at $-78{ }^{\circ} \mathrm{C}$ allowing warming to $\mathrm{rt}$ and then stirred for an additional hour. The reaction was 
quenched by pouring the reaction mixture into aqueous saturated ammonium chloride solution (100 $\mathrm{ml})$. It was then extracted with ethyl acetate $(3 \times 50 \mathrm{ml})$. The combined organic fractions were washed with brine, dried over anhydrous sodium sulfate and concentrated in vacuo. Column chromatography (90:10, hexanes-ethyl acetate) afforded 3-phenylselanylpyrrolidine-1,3-dicarboxylic acid 1-benzyl ester 3-methyl ester 15 as a yellow solid (7.0 g, 77\%), mp. 88-90 ${ }^{\circ} \mathrm{C}$. (Found; $\left[\mathrm{M}+\mathrm{NH}_{4}\right]^{+}, 437.0977$, $\mathrm{C}_{20} \mathrm{H}_{21} \mathrm{NO}_{4} \mathrm{Se}$ requires $\left[\mathrm{M}+\mathrm{NH}_{4}\right]^{+}$437.0974). $v_{\max }\left(\mathrm{KBr}, \mathrm{cm}^{-1}\right)$ 2949, 1726, 1705, 1416, 1468, 1212 and 1104. $\delta_{\mathrm{H}}\left(400 \mathrm{MHz} ; \mathrm{CDCl}_{3}\right) 2.04-2.10\left(1 \mathrm{H}, \mathrm{m}, \mathrm{CH}_{2}\right), 2.30-2.43\left(1 \mathrm{H}, \mathrm{m}, \mathrm{CH}_{2}\right), 3.65\left(3 \mathrm{H}, \mathrm{s}, \mathrm{OCH}_{3}\right)$, 3.50-3.84 (4H, m, $\left.\mathrm{CH}_{2}\right), 5.09-5.18\left(2 \mathrm{H}, \mathrm{m}, \mathrm{CH}_{2}\right), 7.27-7.58(10 \mathrm{H}, \mathrm{m}, \mathrm{ArCH}) . \delta_{\mathrm{C}}\left(100.61 \mathrm{MHz}, \mathrm{CDCl}_{3}\right)$ 33.3 and $34.1\left(\mathrm{CH}_{2}\right), 44.9$ and $45.2\left(\mathrm{CH}_{2}\right), 52.1$ and $52.8(\mathrm{C}), 52.6\left(\mathrm{OCH}_{3}\right), 53.1$ and $53.7\left(\mathrm{CH}_{2}\right), 67.1$ and $67.2\left(\mathrm{CH}_{2}\right), 126.8(\mathrm{C}), 128.0(\mathrm{CH}), 128.1(\mathrm{CH}), 128.6(\mathrm{CH}), 129.2(\mathrm{CH}), 130.0(\mathrm{CH}) 136.8(\mathrm{C})$, $137.8(\mathrm{CH}), 154.8(\mathrm{C})$ and $172.4(\mathrm{C}) ; \mathrm{m} / z$ (EI) $419\left(\mathrm{M}^{+}\right),(100 \%) 417(50), 415(20)$ and $312(60)$.

\section{3-(4-Methoxyphenylcarbamoyl)-3-phenylselanylpyrrolidine-1-carboxylic acid benzyl ester 16:}

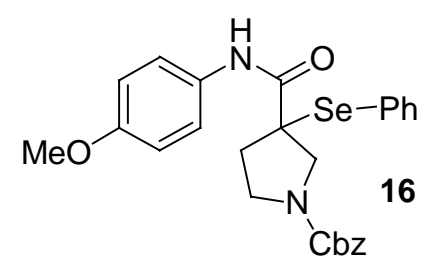

Trimethylaluminium (2 $\mathrm{M}$ in toluene, $3.9 \mathrm{ml}, 7.75 \mathrm{mmol}, 1.2$ equiv.) was added dropwise to the stirred solution of $p$-anisidine ( $0.95 \mathrm{~g}, 7.75 \mathrm{mmol}, 1.2$ equiv.) in dry toluene $(20 \mathrm{ml})$ at $0{ }^{\circ} \mathrm{C}$ under nitrogen. A solution of 3-phenylselanylpyrrolidine-1,3-dicarboxylic acid 1-benzyl ester 3-methyl ester 15 (2.7 g, $6.5 \mathrm{mmol}, 1.0$ equiv.) in dry toluene $(20 \mathrm{ml})$ was added to it at room temperature and the mixture stirred overnight at ambient temperature. It was then cooled and quenched by pouring it into aqueous saturated ammonium chloride solution $(20 \mathrm{ml})$ and extracted with ethyl acetate. The combined organic fractions were washed with brine $(50 \mathrm{ml})$, dried over anhydrous sodium sulfate and concentrated in vacuo to afford the crude product that was purified by column chromatography $(80: 10: 10$, hexanes ethyl acetate - dichloromethane) to afford 3-(4-methoxyphenylcarbamoyl)-3-phenylselanylpyrrolidine1-carboxylic acid benzyl ester 16 as a pale yellow sticky solid (3.2 g, 97\%). (Found; $\left[\mathrm{M}+\mathrm{NH}_{4}\right]^{+}$, 528.1396, $\mathrm{C}_{26} \mathrm{H}_{26} \mathrm{~N}_{2} \mathrm{O}_{4} \mathrm{Se}$ requires $\left[\mathrm{M}+\mathrm{NH}_{4}\right]^{+}$528.1398). $v_{\max }\left(\right.$ film, $\left.\mathrm{cm}^{-1}\right)$ 3327, 3054, 2956, 1699, 1598, 1513, 1423, 1361, 1246, 1108 and 1033. $\delta_{\mathrm{H}}\left(400 \mathrm{MHz} ; \mathrm{CDCl}_{3}\right) 2.06-2.12\left(1 \mathrm{H}, \mathrm{m}, \mathrm{CH}_{2}\right), 2.48-$ $2.65\left(1 \mathrm{H}, \mathrm{m}, \mathrm{CH}_{2}\right), 3.63-3.97\left(4 \mathrm{H}, \mathrm{m}, \mathrm{CH}_{2}\right), 3.77\left(3 \mathrm{H}, \mathrm{s}, \mathrm{OCH}_{3}\right), 5.12-5.22\left(2 \mathrm{H}, \mathrm{m}, \mathrm{CH}_{2}\right), 6.68-6.91$ $(2 \mathrm{H}, \mathrm{m}, \mathrm{ArCH}), 7.25-7.39(10 \mathrm{H}, \mathrm{m}, \mathrm{ArCH}), 7.53-7.59(2 \mathrm{H}, \mathrm{m}, \mathrm{ArCH}), 8.20(1 \mathrm{H}, \mathrm{s}, \mathrm{NH}) ; \delta_{\mathrm{C}}(100.61$ $\left.\mathrm{MHz}, \mathrm{CDCl}_{3}\right) 34.2$ and $34.8\left(\mathrm{CH}_{2}\right), 45.4$ and $45.9\left(\mathrm{CH}_{2}\right), 54.1$ and $54.8\left(\mathrm{CH}_{2}\right), 55.8\left(\mathrm{OCH}_{3}\right), 56.3$ and 57.4 (C), $67.4\left(\mathrm{CH}_{2}\right), 114.5$ and $114.5(\mathrm{CH}), 122.2$ and $122.3(\mathrm{CH}), 126.9(\mathrm{C}), 128.1$ and $128.3(\mathrm{CH})$, $128.4(\mathrm{CH}), 128.8(\mathrm{CH}), 129.8(\mathrm{CH}), 130.0(\mathrm{CH}), 130.9(\mathrm{C}), 136.9(\mathrm{C}), 137.0(\mathrm{CH}), 155.0$ and 155.1 (C), $157.0(\mathrm{C}), 169.3$ and $169.4(\mathrm{C}) ; \mathrm{m} / \mathrm{z}$ (EI) $510\left(\mathrm{M}^{+}\right),(100 \%) 508(60), 442$ (50) and 403 (30). 
3-[Benzyl-(4-methoxyphenyl)carbamoyl]-3-phenylselanylpyrrolidine-1-carboxylic acid benzyl ester 17:

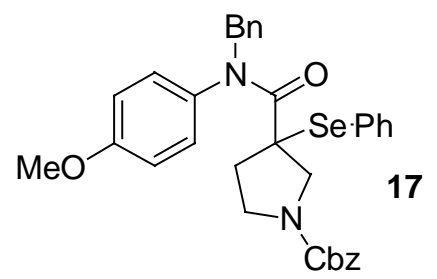

Potassium tert-butoxide ( $0.18 \mathrm{~g}, 1.47 \mathrm{mmol}, 1.5$ equiv.) was dissolved in dry tetrahydrofuran (20 ml) under nitrogen and cooled to 0 ( ${ }^{\circ} \mathrm{C}$. A solution of 3-(4-methoxyphenylcarbamoyl)-3phenylselanylpyrrolidine-3-carboxylic acid benzyl ester 16 (0.5 g, 0.98 mmol, 1.0 equiv.) in dry tetrahydrofuran $(20 \mathrm{ml})$ was added drop-wise to it and the mixture refluxed for $4 \mathrm{~h}$. Benzyl bromide ( $0.18 \mathrm{ml}, 1.47 \mathrm{mmol}, 1.5$ equiv.) was then added and the reaction mixture refluxed for an overnight period. It was cooled and quenched with aqueous saturated ammonium chloride $(50 \mathrm{ml})$ and extracted with ethyl acetate $(3 \times 40 \mathrm{ml})$. The combined organic fractions were washed with brine $(50 \mathrm{ml})$, dried over anhydrous sodium sulfate and concentrated in vacuo to afford the crude product that was purified by column chromatography (80:10:10, hexanes- ethyl acetate-dichloromethane) afforded 3-[benzyl-(4methoxyphenylcarbamoyl)-3-phenylselanylpyrrolidine-3-carboxylic acid benzyl ester $\mathbf{1 7}$ as a pale yellow sticky solid $\left(0.5 \mathrm{~g}, 85 \%\right.$ ). (Found; $[\mathrm{M}+\mathrm{H}]^{+}, 601.1600, \mathrm{C}_{26} \mathrm{H}_{26} \mathrm{~N}_{2} \mathrm{O}_{4} \mathrm{Se}$ requires $[\mathrm{M}+\mathrm{H}]^{+}$ 601.1606). $v_{\max }\left(\right.$ film, $\left.\mathrm{cm}^{-1}\right) 2937,1704,1632,1510,1419,1250,1135$ and $1106 . \delta_{\mathrm{H}}\left(400 \mathrm{MHz} ; \mathrm{CDCl}_{3}\right)$ 1.27-1.34 (1H, m, CH $\left.\mathrm{CH}_{2}\right)$ 2.01-2.18 $\left(1 \mathrm{H}, \mathrm{m}, \mathrm{CH}_{2}\right), 2.92-3.07\left(1 \mathrm{H}, \mathrm{m}, \mathrm{CH}_{2}\right), 3.35-3.56\left(3 \mathrm{H}, \mathrm{m}, \mathrm{CH}_{2}\right)$, $3.80\left(3 \mathrm{H}, \mathrm{s}, \mathrm{OCH}_{3}\right), 4.76-5.00\left(2 \mathrm{H}, \mathrm{m}, \mathrm{CH}_{2}\right), 5.03-5.14(2 \mathrm{H}, \mathrm{m}, \mathrm{ArH}), 6.81-6.85(2 \mathrm{H}, \mathrm{m}, \mathrm{ArH}), 7.11-$ $7.41(15 \mathrm{H}, \mathrm{m}, \mathrm{ArH}), 7.48-7.50(2 \mathrm{H}, \mathrm{m}, \mathrm{ArH}) . \delta_{\mathrm{C}}\left(100.61 \mathrm{MHz}, \mathrm{CDCl}_{3}\right) 34.9$ and $35.6\left(\mathrm{CH}_{2}\right), 44.5$ and $45.3\left(\mathrm{CH}_{2}\right), 54.2$ and $54.5\left(\mathrm{CH}_{2}\right), 55.0$ and $55.7(\mathrm{C}), 55.8\left(\mathrm{OCH}_{3}\right), 56.0$ and $56.1\left(\mathrm{CH}_{2}\right), 67.0\left(\mathrm{CH}_{2}\right)$, 114.4 and $114.5(\mathrm{CH}), 127.8(\mathrm{C}), 127.9(\mathrm{CH}), 128.0(\mathrm{CH}), 128.1(\mathrm{CH}), 128.2(\mathrm{CH}), 128.7(\mathrm{CH}), 128.8$ $(\mathrm{CH}), 129.3$ and $129.4(\mathrm{CH}), 129.7$ and $129.8(\mathrm{CH}), 129.9(\mathrm{CH}), 131.9(\mathrm{CH}), 134.0$ and $134.2(\mathrm{C})$, $137.0(\mathrm{C}), 137.6(\mathrm{C}), 137.8$ and $137.9(\mathrm{CH}), 154.9(\mathrm{C}), 159.7(\mathrm{C}), 170.9$ and $171.1(\mathrm{C}) ; \mathrm{m} / \mathrm{z}\left(\mathrm{CI}^{+}\right) 618$ $\left(\mathrm{MNH}_{4}^{+}\right)(80 \%), 601(100), 599(55)$ and $598(20)$.

1-Benzyl-5-methoxy-2-oxo-1,2-dihydro-spiro[indole-3,3'-pyrrolidine]-1'- carboxylic acid benzyl ester 19:

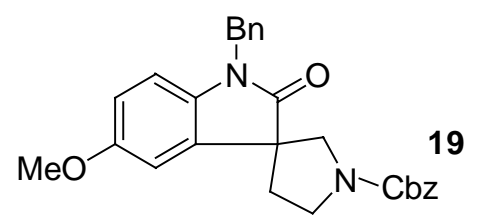

A solution of 3-[benzyl-(4-methoxyphenyl)carbamoyl]-3-phenylselanylpyrrolidine-3-carboxylic acid benzyl ester 19 (0.071 g, $0.118 \mathrm{mmol}, 1.0$ equiv.) in benzene $(7 \mathrm{ml})$ was purged with argon and the heated to reflux for an hour. It was cooled to room temperature and to it DEPO $(0.25 \mathrm{~g}, 2.37 \mathrm{mmol}$, 20.0 equiv.) was added under argon atmosphere and again heated to reflux. AIBN (0.02 g, $0.142 \mathrm{mmol}$, 1.2 equiv.) was added under argon atmosphere and the mixture continued to stir at reflux temperature for $48 \mathrm{~h}$. AIBN (0.02 g, $0.142 \mathrm{mmol}, 1.2$ equiv.) was added with an interval of $8 \mathrm{~h}$ (total initiator added 
$=3.6$ equiv.). After 2 days, the reaction mixture was cooled to room temperature and the solvent removed in vacuo. The residue was dissolved in diethyl ether and washed with aqueous sodium hydroxide solution $(2 \mathrm{M}, 20 \mathrm{ml})$ and extracted with diethyl ether $(2 \times 20 \mathrm{ml})$. The combined organic fractions were washed with brine $(40 \mathrm{ml})$, dried over anhydrous sodium sulfate and concentrated in vacuo. Purification by column chromatography (60:20:20, hexanes-ethyl acetate-dichloromethane) afforded 1-benzyl-5-methoxy-2-oxo-1,2-dihydro-spiro[indole-3,3'-pyrrolidine]-1'-carboxylic acid benzyl ester 19 as a pale yellow sticky solid (0.045 g, 85\%). (Found; $[\mathrm{M}+\mathrm{H}]^{+}, 443.1967, \mathrm{C}_{27} \mathrm{H}_{26} \mathrm{~N}_{2} \mathrm{O}_{4}$ requires $[\mathrm{M}+\mathrm{H}]^{+}$443.1965). $v_{\max }\left(\right.$ film, $\left.\mathrm{cm}^{-1}\right) 3008,1701,1603,1512,1496,1455,1424,1346$ and 1177. $\delta_{\mathrm{H}}\left(400 \mathrm{MHz} ; \mathrm{CDCl}_{3}\right) 2.07-2.18\left(1 \mathrm{H}, \mathrm{m}, \mathrm{CH}_{2}\right), 2.46-2.54\left(1 \mathrm{H}, \mathrm{m}, \mathrm{CH}_{2}\right), 3.66-3.74\left(1 \mathrm{H}, \mathrm{m}, \mathrm{CH}_{2}\right)$, $3.77\left(3 \mathrm{H}, \mathrm{s}, \mathrm{OCH}_{3}\right), 3.79-4.01\left(3 \mathrm{H}, \mathrm{m}, \mathrm{CH}_{2}\right), 4.86-4.94\left(2 \mathrm{H}, \mathrm{m}, \mathrm{CH}_{2}\right), 5.11-5.21\left(2 \mathrm{H}, \mathrm{m}, \mathrm{CH}_{2}\right), 6.63-$ $6.65(1 \mathrm{H}, \mathrm{d}, J$ 8.5 $\mathrm{ArH}), 6.69-6.71(1 \mathrm{H}, \mathrm{dd}, J 8.5,2.4, \mathrm{ArH})$ and 6.79-6.81 (1H, d, $J 2.2, \mathrm{ArH}), 7.24-$ 7.44(10H, m, ArH). $\delta_{\mathrm{C}}\left(100.61 \mathrm{MHz}, \mathrm{CDCl}_{3}\right) 35.8$ and $36.6\left(\mathrm{CH}_{2}\right), 44.1$ and $44.2\left(\mathrm{CH}_{2}\right), 45.5$ and 46.0 (C), 52.5 and $53.4\left(\mathrm{CH}_{2}\right), 54.4$ and $54.8\left(\mathrm{CH}_{2}\right), 56.0\left(\mathrm{OCH}_{3}\right), 67.2\left(\mathrm{CH}_{2}\right), 109.9(\mathrm{CH}), 110.4$ and 110.5 $(\mathrm{CH}), 112.5$ and $112.6(\mathrm{CH}), 127.4(\mathrm{CH}), 127.9(\mathrm{CH}), 128.0$ and $128.1(\mathrm{CH}), 128.5$ and $128.7(\mathrm{CH})$, $129.0(\mathrm{CH}), 133.3(\mathrm{C}), 133.8(\mathrm{C}), 135.5$ and $135.9(\mathrm{C}), 136.8(\mathrm{C}), 154.9(\mathrm{C}), 156.5$ and $156.6(\mathrm{C})$, 177.2 and $177.6(\mathrm{C}) ; \mathrm{m} / z\left(\mathrm{EI}^{+}\right) 442\left(\mathrm{M}^{+}\right)$(60\%), 351 (20), $307(45), 278(40), 265(100)$ and 234 (20).

\section{3-[Benzyl-(4-methoxyphenyl)-carbamoyl] pyrrolidine-1-carboxylic acid benzyl ester 18:}

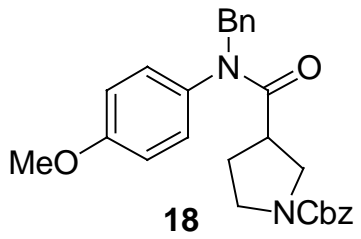

A solution of 1-EPHP $\left(0.6 \mathrm{~g}, 3.34 \times 10^{-3} \mathrm{M}, 10.0\right.$ equiv) and 3-[benzyl-(4-methoxyphenyl)carbamoyl]3-phenylselanylpyrrolidine-3-carboxylic acid benzyl ester, 17, (0.2 g, 3.34 x 10-4 M, 1.0 equiv) in dry toluene $(10 \mathrm{ml})$ was purged with argon for an hour and the heated to $80{ }^{\circ} \mathrm{C}$. AIBN $\left(0.06 \mathrm{~g}, 4.0 \times 10^{-4} \mathrm{M}\right.$, 1.2 equiv) was added and the mixture continued to stir at $80{ }^{\circ} \mathrm{C}$. After $5 \mathrm{~min}$ of heating, the mixture turned green at which point the reflux was stopped and cooled to room temperature. Solvent was removed in vacuo and the crude product was purified by column chromatography (ethyl acetate: dichloromethane: petroleum ether/1:4:5) to afford the reduced product 18 3-[benzyl-(4methoxyphenyl)-carbamoyl] pyrrolidine-1-carboxylic acid benzyl ester $(0.126 \mathrm{~g}, 85 \%)$ as a pale yellow sticky solid. (Found; $[\mathrm{M}+\mathrm{H}]^{+}, 445.2122, \mathrm{C}_{27} \mathrm{H}_{28} \mathrm{~N}_{2} \mathrm{O}_{4}$ requires $\left.[\mathrm{M}+\mathrm{H}]^{+} 445.2119\right) . v_{\max }\left(\right.$ Film, $\mathrm{cm}^{-1}$ ) $3007,1695,1694,1511,1422,1410,1250,1121$ and $1028 . \delta_{\mathrm{H}}\left(400 \mathrm{MHz} ; \mathrm{CDCl}_{3}\right) 1.87-1.90(1 \mathrm{H}, \mathrm{m}$, $\left.\mathrm{CH}_{2}\right), 2.10-2.33\left(1 \mathrm{H}, \mathrm{m}, \mathrm{CH}_{2}\right), 2.89-2.93(1 \mathrm{H}, \mathrm{m}, \mathrm{CH}), 3.23-3.25\left(1 \mathrm{H}, \mathrm{m}, \mathrm{CH}_{2}\right), 3.43-3.68(3 \mathrm{H}, \mathrm{m}$, $\left.\mathrm{CH}_{2}\right), 3.80\left(3 \mathrm{H}, \mathrm{s}, \mathrm{OCH}_{3}\right), 4.80-4.93\left(2 \mathrm{H}, \mathrm{m}, \mathrm{CH}_{2}\right), 5.12\left(2 \mathrm{H}, \mathrm{s}, \mathrm{CH}_{2}\right), 6.83-6.89(4 \mathrm{H}, \mathrm{m}, \mathrm{ArCH}), 7.17-$ $7.19(2 \mathrm{H}, \mathrm{m}, \mathrm{ArCH})$ and 7.26-7.36 (8H, m, $\mathrm{ArCH}) . \delta_{\mathrm{C}}\left(100.61 \mathrm{MHz}, \mathrm{CDCl}_{3}\right) 29.4$ and $29.7\left(\mathrm{CH}_{2}\right), 40.7$ and $41.7(\mathrm{CH}), 45.6$ and $46.0\left(\mathrm{CH}_{2}\right), 49.1$ and $49.8\left(\mathrm{CH}_{2}\right), 53.4\left(\mathrm{CH}_{2}\right), 55.5\left(\mathrm{OCH}_{3}\right), 66.7\left(C \mathrm{H}_{2}\right), 114.9$ $(C H), 127.5(C H), 127.9(C H), 128.5(C H), 128.9$ and $129.0(C H), 129.5(C H), 134.4(C), 137.0(C)$, 
$137.4(C), 154.6(C), 159.3(C), 172.4$ and $172.6(C) ; \mathrm{m} / z\left(\mathrm{CI}^{+}\right) 462\left(\mathrm{MNH}_{4}^{+}\right)(70 \%), 445(100), 311$ (20), $214(20)$ and 108 (15). 